\title{
Microcomputed tomography of the femur of diabetic rats: alterations of trabecular and cortical bone microarchitecture and vasculature-a feasibility study
}

David Zeitoun ${ }^{1 *}$ (D), Guavri Caliaperoumal ${ }^{2}$, Morad Bensidhoum², Jean Marc Constans ${ }^{3}$, Fani Anagnostou ${ }^{2}$ and Valérie Bousson ${ }^{1}$

\begin{abstract}
Background: To better understand bone fragility in type 2 diabetes mellitus and define the contribution of microcomputed tomography (micro-CT) to the evaluation of bone microarchitecture and vascularisation, we conducted an in vitro preliminary study on the femur of Zucker diabetic fatty (ZDF) rats and Zucker lean (ZL) rats. We first analysed bone microarchitecture, then determined whether micro-CT allowed to explore bone vascularisation, and finally looked for a link between these parameters.

Methods: Eight ZDF and six ZL rats were examined for bone microarchitecture (group 1), and six ZDF and six ZL rats were studied for bone vascularisation after Microfil ${ }^{\oplus}$ perfusion which is a radiopaque casting agent (group 2). In group 1, we used micro-CT to examine the trabecular and cortical bone microarchitecture of the femoral head, neck, shaft, and distal metaphysis. In group 2, micro-CT was used to study the blood vessels in the head, neck, and distal metaphysis.

Results: Compared to ZL rats, the ZDF rats exhibited significantly lower trabecular bone volume and number and higher trabecular separation in the three locations $(p=0.02, p=0.02, p=0.003)$. Cortical porosity was significantly higher in the ZDF rats at the neck and shaft $(p=0.001$ and $p=0.005)$. We observed a dramatically poorer bone vascularisation in the femur of ZDF rats, especially in distal metaphysis $(p<0.047)$.

Conclusions: Micro- $C T$ demonstrated not only significant alterations in the bone microarchitecture of the femurs of ZDF rats, but also significant alterations in bone vascularisation. Further studies are required to demonstrate the causal link between poor vascularisation and impaired bone architecture.
\end{abstract}

Keywords: Bone and bones, Diabetes mellitus (type 2), Rats (Zucker), Vascular diseases, X-ray microtomography

\section{Key points}

- Microcomputed tomography (micro-CT) is a promising tool to study bone vascularisation

- Trabecular and cortical bone quantity and microarchitecture were altered in the femur of Zucker diabetic fatty (ZDF) rats
- In ZDF rats, an impaired bone architecture is associated to a poor bone vascularisation

- The main region of interest for studying architecture and vasculature changes in the femur of ZDF rats is the distal metaphysis

\section{Background}

Type 2 diabetes mellitus (T2DM) is a disease whose worldwide incidence is increasing [1]. Morbidity and mortality of this disease are mainly due to vascular complications, but more recently, increased risk of fractures has emerged as a serious complication in patients with long-standing or

\footnotetext{
* Correspondence: dzeitoun2@gmail.com

${ }^{1}$ Centre hospitalier Lariboisière, Hopital Lariboisière, Service de radiologie

ostéo-articulaire, 2 rue Ambroise Paré, 75010 Paris, France

Full list of author information is available at the end of the article
} 
poorly controlled disease $[2,3]$. This increased risk of fractures has a distinct propensity for the proximal end of the femur [4-7] and vertebrae [8] and is problematic because patients with T2DM exhibit compromised bone fracture healing and poorer outcomes after fracture [9-11].

A paradox of this clinical syndrome is that increased risk of bone fracture is evident despite higher body weight and normal-to-high areal bone mineral density [12], which are generally associated with reduced bone fracture risk. The increased risk of bone fracture is also not explained by a higher frequency of falls among T2DM patients [13]. Several studies using high-resolution peripheral quantitative computed tomography in T2DM patients found that the microarchitecture of the trabecular bone tissue had been preserved but cortical porosity had increased and bone quality was impaired [14-16]. Definitive elucidation of the pathogenesis of these abnormalities will require further studies.

The use of animal models is essential in research pertaining to T2DM. Zucker diabetic fatty (ZDF) rats constitute a well-documented model for T2DM because these animals have a mutation that is responsible for leptin receptor deficiency and develop insulin-resistant hyperglycaemia and metabolic syndrome at the age of 9 weeks [17]. The ZDF model was used to demonstrate a significant association between T2DM and decreased BMD, trabecular bone volume (Tb.BV), and cortical thickness (Ct.Th) in the vertebrae and in the distal metaphyses of femurs [18-21]. The ZDF model was also used to test several hypotheses, such as an increase in non-enzymatic glycation [15] [22-24] and reduction in bone turnover [25-27], regarding the impaired bone quality observed in T2DM. However, to the best of our knowledge, there is no data available in the literature on the proximal femur, which is a determinant location for the fragility fractures, or on cortical porosity, which is a determinant factor for bone strength. Finally, although microangiopathy is involved in most T2DM complications, bone vascularisation and its potential impaction with bone microstructure remains poorly documented.

To better understand bone fragility in T2DM and define the contribution of microcomputed tomography (microCT) to the evaluation of bone microarchitecture and vascularisation, we conducted an in vitro preliminary study on ZDF and control rats. We first analysed trabecular and cortical bone microarchitecture of the proximal, mid-diaphyseal, and distal femur, then determined whether micro-CT allowed to explore bone vascularisation of the femurs, and finally looked for a link between microstructure and vascularisation parameters.

\section{Methods}

\section{Animal model}

The experimental procedures involving the rats used in the present study were approved by the Ethics Committee of the University Paris Diderot (No. 01610.01/S69), Paris, France. Twenty-six rats were studied: 14 ZDF rats and 12 age-matched Zucker lean (ZL) rats as controls. Among them, $8 \mathrm{ZDF}$ and $6 \mathrm{ZL}$ rats were investigated for bone microarchitecture (group 1), while $6 \mathrm{ZDF}$ and $6 \mathrm{ZL}$ rats were studied for bone vascularisation (group 2). The animals were maintained under standard animal laboratory conditions with a light-dark cycle of $12 \mathrm{~h}$ and a temperature of $20-21^{\circ} \mathrm{C}$. The animals had free access to water and were fed with high fat and high carbohydrate foods. At 21 weeks, blood sugar level and alkaline phosphate, fructosamine, and calcium blood level were determined, and the animal body weight was measured. At 24 weeks, the rats of group 2 (6 ZDF and $6 \mathrm{ZL}$ rats) received an injection of Microfil $^{\circ}$ (Microfil red compound MV 130, Flow Tech Inc., Carver, MA, USA) which is a radiopaque casting agent. At that time, the 26 rats were euthanised and their right femur excised for analyses.

\section{Image acquisition}

Micro-CT images were acquired using a Skyscan 1172 micro-CT (Bruker, Hambourg, Germany), at three sites of the excised femurs: proximal and distal ends, and shaft, of the femur of both ZDF rats $(n=8)$ and ZL rats $(n=6)$. The micro-CT images were acquired at the following setting: $80 \mathrm{kVp}, 100 \mathrm{mAs}$, matrix $2000 \times 1336$, exposure time $110 \mathrm{~ms}$, filter $\mathrm{Al} 0.5$, and voxel size $6 \mu \mathrm{m}$.

\section{Image analysis}

Image analysis was performed using the CTAn software (Bruker, Hambourg, Germany).

\section{Definition of the volume of interest (VOI) for trabecular bone and cortical bone}

Trabecular bone was analysed in the head, neck, and distal metaphysis of the femurs. Cortical bone was analysed in the neck and shaft. VOIs were determined using semiautomatic and robust segmentation techniques. We obtained the VOIs from several selected images and the software then interpolated between them. Details regarding image selection for each VOI were as follows:

- For the head, the first axial image in the head served as a reference; we moved forward by 100 images and plotted the VOI on the following 300 images.

- For the neck, images were first reoriented along the direction of the neck axis and the first image in the neck was chosen as a reference; we moved forward by 100 images for trabecular bone and 200 images for cortical bone; the VOI extended over 100 images for cortical and trabecular bone.

- For the shaft, the reference section was the growth plate; we moved forward by 500 images and plotted 
the VOI between the endosteum and periosteum on 100 images.

- For the distal metaphysis, the growth plate served as a reference; we moved forward by 300 images to plot the VOI within the endosteum; the VOI extended over 300 images.

\section{Thresholding}

Trabecular and cortical bone were investigated using global thresholding. For each trabecular bone specimen, the threshold was fixed at the minimal intensity between the two curves of the histograms representing bone and bone marrow. This threshold allowed for separation of the bone from the bone marrow. Due to the small pore surface area and partial volume effects in cortical bone, only one curve representing bone tissue was obtained. For this reason, the threshold for each specimen was placed on the minimum intensity of the bone tissue, before the beginning of the curve. This threshold allowed us to separate the pores from the bone tissue.

\section{Assessed variables}

The trabecular bone variables assessed were as follows: trabecular bone volume (Tb.BV), trabecular bone percentage (Tb.BV/TV), trabecular thickness (Tb.Th), trabecular number (Tb.N), trabecular separation (Tb.Sp), index of connectivity (Conn.D), structural model index (SMI), and volumetric bone mineral density (vBMD). The cortical bone variables assessed were as follows: cortical bone percentage (Ct.BV/TV), cortical surface (Ct.Ar), cortical perimeter (Ct.P), cortical thickness (Ct.Th), cortical porosity (Ct.Po), and volumetric bone mineral density (vBMD).

\section{Microfil $^{\circledR}$ vascular injection and micro-CT parameters of bone vasculature}

Bone vascularisation was determined using 12 rats (6 ZDF rats and $6 \mathrm{ZL}$ rats). In order to obtain three-dimensional views of the rat vascular network using micro-CT, Microfil $^{\circ}$ (MicrofilTM red compound MV 130, Flow Tech Inc., Carver, MA, USA) [28] was injected.

The procedure started with anaesthetising the rats using $100 \mu \mathrm{L}$ of ketamine (100 mg/mL; Pfizer, NYC, USA) and $50 \mu \mathrm{L}$ of Xyzalin $(20 \mathrm{mg} / \mathrm{mL}$, Bayer, Leverkusen, Germany). The skin from the thorax and the rib cage of each animal was incised to access the heart, and $0.3 \mathrm{~mL}$ heparin $(5.000$ $\mathrm{UI} / \mathrm{mL}$ ) was injected directly in the left ventricle. After 10 min, the left ventricle was catheterised using a $22 \mathrm{G}$ canula and the right atrium was cut to remove the contained blood. First, animals were perfused with isotonic saline $(200 \mathrm{~mL})$ containing heparin $(100 \mathrm{UI} / \mathrm{mL})$ to rinse the blood vessels. Animals were then perfused via the intracardiac canula with $50 \mathrm{~mL}$ of Microfil $^{\circ}$ (prepared according to the manufacturer's instructions using $20 \mathrm{~mL}$ of Microfil ${ }^{\circ}$, $25 \mathrm{~mL}$ of the specific diluent, and $5 \mathrm{~mL}$ of the specific curing agent). The pressure during filling was of approximately $120 \mathrm{mmHg}$ in order to force Microfil ${ }^{\circ}$ into the capillary networks without extravasation into the surrounding tissue. This injection was considered successful when perfect opacification of the digestive tract capillaries was observed. Such a result was obtained only in seven of the 12 rats tested (4 ZDF and $3 \mathrm{ZL}$ rats). The five rats with imperfect opacification were used to determine the decalcification and micro-CT protocols. Due to the similar micro-CT values between opacified vessels and bone, the excised femurs had to be decalcified before micro-CT analysis in order to assess the presence of vessels [29]. The parameters used for Micro-CT image acquisition were $80 \mathrm{kVp}, 100 \mathrm{mAs}$, matrix $4.000 \times 2.272$, time exposure 280 $\mathrm{ms}$, no filters, and voxel size $3 \mu \mathrm{m}$.

The VOIs for the vessel analyses were identical to those defined for bone microarchitecture evaluation. A global thresholding technique was used for segmentation. For each specimen, the threshold was placed at the end of the curve that separates bone marrow and blood vessels. The following data were obtained using CTAn (Bruker, Hambourg, Germany): vessel volume (VV), vessel percentage (VV/TV \%), vessel thickness (V.Th), vessel number (V.N), and vessel separation (V.Sp).

\section{Statistical analysis}

All experimental data were compiled as means and standard deviations (mean \pm standard deviation) and reported separately for each group of rats. Comparisons of the variables of bone microarchitecture and bone vascularisation between ZDF and ZL rats were carried out using a non-parametric test (Mann-Whitney $U$ ). All tests were two-sided with a significance level equal to 0.05 and analysis were made using SPPS for Windows (v.10, SPSS Inc., Chicago, IL, USA).

\section{Results}

The 24-week-old ZDF and ZL rats had an average weight of $439.4 \pm 16.35 \mathrm{~g}$ and $387.2 \pm 10.35 \mathrm{~g}$, respectively. The ZDF rats had higher blood serum glucose levels than the ZL rats $(5.88 \pm 0.17$ versus $2.33 \pm 0.15$ ( $p$ $<0.001)$ ). In addition, the ZDF rats also had significantly increased levels of fructosamine $(p=0.001)$, alkaline phosphatase $(p=0.005)$, and calcium $(p=0.001)$.

\section{Bone microarchitecture in ZDF and ZL rats}

The results and comparisons of trabecular bone microarchitecture between ZDF and ZL rats are shown in Table 1. Compared to ZL rats, the trabecular bone of ZDF rats was significantly different. The ZDF rats had significantly lower Tb.BV values in the head $(p=0.003)$, neck ( $p$ $=0.02)$, and distal metaphysis $(p=0.001)$ (Fig. 1). Tb.N was significantly lower, and Tb.Sp significantly higher in the head $(p=0.02)$, neck $(p=0.02)$, and distal metaphysis 
Table 1 Analysis of trabecular bone

\begin{tabular}{|c|c|c|c|}
\hline & ZL rats & ZDF rats & $p$ \\
\hline \multicolumn{4}{|l|}{ Femoral head } \\
\hline Tb.BV/TV (\%) & $81.01 \pm 1.7$ & $69.35 \pm 4.73$ & 0.003 \\
\hline SMI & $-9.74 \pm 2.4$ & $-5.49 \pm 1.6$ & 0.02 \\
\hline Tb.N (1/mm) & $8.38 \pm 0.86$ & $7.87 \pm 1.078$ & 0.04 \\
\hline Tb.Sp (mm) & $0.056 \pm 0.006$ & $0.07 \pm 0.014$ & 0.02 \\
\hline Tb.Th (mm) & $0.10 \pm 0.017$ & $0.08 \pm 0.013$ & 0.005 \\
\hline Conn.D $\left(1 / \mathrm{mm}^{3}\right)$ & $1270 \pm 197$ & $2237 \pm 646$ & 0.07 \\
\hline \multicolumn{4}{|l|}{ Femoral neck } \\
\hline Tb.BV/TV (\%) & $46.9 \pm 4.61$ & $35.37 \pm 6.67$ & 0.02 \\
\hline SMI & $0.89 \pm 0.48$ & $1.26 \pm 0.22$ & 0.04 \\
\hline Tb.N (1/mm) & $6.07 \pm 0.62$ & $4.90 \pm 0.92$ & 0.02 \\
\hline Tb.Sp (mm) & $0.10 \pm 0.02$ & $0.13 \pm 0.03$ & 0.02 \\
\hline Tb.Th (mm) & $0.077 \pm 0.008$ & $0.07 \pm 0.004$ & 0.22 \\
\hline Conn.D $\left(1 / \mathrm{mm}^{3}\right)$ & $1320 \pm 458$ & $1312 \pm 675$ & 0.34 \\
\hline vBMD & $0.57 \pm 11$ & $0.49 \pm 0.06$ & 0.14 \\
\hline \multicolumn{4}{|l|}{ Distal metaphysis } \\
\hline Tb.BV/TV (\%) & $29.56 \pm 4.61$ & $19.38 \pm 4.07$ & 0.001 \\
\hline SMI & $0.81 \pm 0.21$ & $1.18 \pm 0.18$ & 0.008 \\
\hline Tb.N (1/mm) & $4.60 \pm 0.62$ & $3.25 \pm 0.73$ & 0.005 \\
\hline Tb.SP (mm) & $0.15 \pm 0.03$ & $0.26 \pm 0.05$ & 0.003 \\
\hline Tb.Th (mm) & $0.06 \pm 0.004$ & $0.05 \pm 0.003$ & 0.08 \\
\hline Conn.D $\left(1 / \mathrm{mm}^{3}\right)$ & $1176 \pm 348$ & $747 \pm 437$ & 0.05 \\
\hline vBMD & $0.39 \pm 0.04$ & $0.27 \pm 0.05$ & 0.003 \\
\hline
\end{tabular}

Data are mean \pm standard deviation. Conn.D index of connectivity, Tb.BV/TV trabecular bone volume percentage, $T b . N$ trabecular number, $T b . S p$ trabecular separation, $T b$.Th trabecular thickness, VBMD volumetric bone mineral density, ZDF Zucker diabetic fatty, ZL Zucker lean

$(p=0.003)$ of the ZDF rats. Tb.Th was significantly lower in the ZDF rats compared to the respective values obtained from the heads of ZL rats $(p=0.005)$, but this was not significant in the neck nor in the distal metaphysis (Fig. 2). SMI was significantly lower in the head, neck, and distal metaphysis of the ZDF rats ( $p$ $=0.02 ; p=0.04 ; p=0.008)$. Conn. $\mathrm{D}$ was not significantly different in the two groups. Trabecular vBMD was significantly lower in the ZDF rats only in the distal metaphysis $(p=0.003)$.

The results and comparisons of cortical bone microarchitecture between $\mathrm{ZDF}$ and $\mathrm{ZL}$ rats are shown in Table 2. Assessment of the cortical bone in the neck and shaft of the femurs also revealed significant differences of several microarchitecture parameters between the two groups. Ct.BV/TV was lower in the ZDF rats, both in the neck $(p=0.001)$ and shaft $(p=0.005)$. Ct.Po was higher in the ZDF rats in the neck $(5.15 \pm 1.7$ versus $3.07 \pm 0.81 ; p=0.001)$ and shaft $(2.14 \pm 0.54$ versus $3.04 \pm 0.99 ; p=0.005$ (Fig. 3). Ct.P,
Ct.Th, and cortical vBMD were not significantly different between the two groups of rats.

\section{Bone vasculature parameters in ZDF and ZL rats}

The results and comparisons of vascularisation parameters between ZDF and ZL rats are shown in Table 3. Compared to results from the ZL rats, the V.V was significantly $(p<$ $0.05)$ lower in the ZDF rats in the neck $(0.012 \pm 0.02$ versus $0.001 \pm 0.008)$ and in the distal metaphysis $(0.40 \pm 0.12$ versus $1.12 \pm 0.32)$. There were also significantly $(p=0.047)$ lower VV/TV in the neck and distal metaphysis $(p=0.047)$ of ZDF rats compared to results obtained from the ZL rats (Fig. 4). The V.Nb values in the neck and in the distal metaphysis of the ZDF versus the ZL rats were significantly lower $(0.30 \pm 0.06$ versus $0.64 \pm 0.07$, respectively, $p=0.047$ and $0.19 \pm 0.06$ versus $0.53 \pm 014$, respectively, $p=0.047$ ) V.Th values were not significantly different between the two groups.

\section{Discussion}

The objective of our study was to use micro-CT to compare trabecular and cortical bone microarchitecture and bone vasculature of the femur of ZDF 24-week rats as a model of long standing diabetic conditions to that of their ZL littermates as controls. The ZDF rats are an established model of T2DM which has been used to examine musculoskeletal complications such as bone fragility [18]. Micro-CT enables a separate evaluation of the cortical and trabecular bone compartments; it has also demonstrated its usefulness in the study of bone vascularisation in healthy rats [30-33].

We observed a significantly lower vBMD in the trabecular compartment of the distal metaphysis of the ZDF rat femurs. Other studies using ZDF rats $[20,21]$ reported that $\mathrm{vBMD}$ varies non-linearly with respect to anatomical location and that the distal femoral metaphysis was the region with the largest vBMD reduction. vBMD, as measured by micro-CT, is an apparent mineral density; it reflects both the amount of bone (bone volume fraction) and the degree of bone mineralisation. Since the degree of bone tissue mineralisation changes very little, vBMD changes mainly reflect changes in the bone volume fraction. Our significantly lower vBMD in the distal diaphysis was clearly related to a significantly lower trabecular bone volume and trabecular number.

Indeed, our study showed a significantly lower trabecular bone volume in the femurs of ZDF rats compared with those of $\mathrm{ZL}$ rats; this was observed in the three VOIs located into the head, neck, and distal metaphysis. This finding may be related to the significant differences observed in trabecular number (lower) and spacing (higher) in the ZDF rats compared to the ZL rats. The trabecular thickness was significantly lower only in the femur head of ZDF compared with ZL rats. 

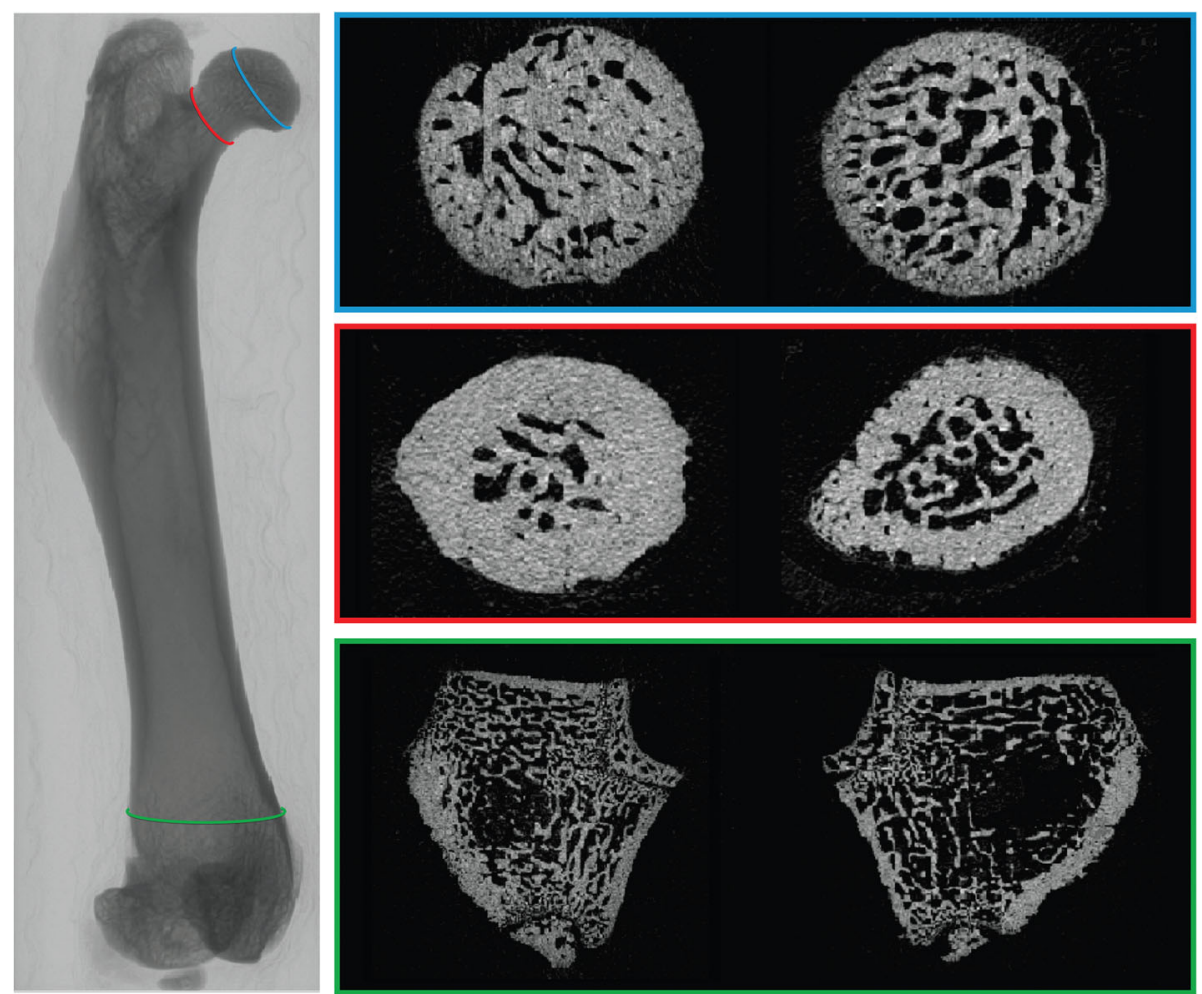

Fig. 1 Comparison of trabecular bone between a Zucker lean rat (ZL, left) and a Zucker diabetic fatty rat (ZDF, right). Axial images of the femoral head, neck, and distal metaphysis. Decreased bone volume and trabecular number in ZDF rats compared to ZL rats, in the three volumes of interest
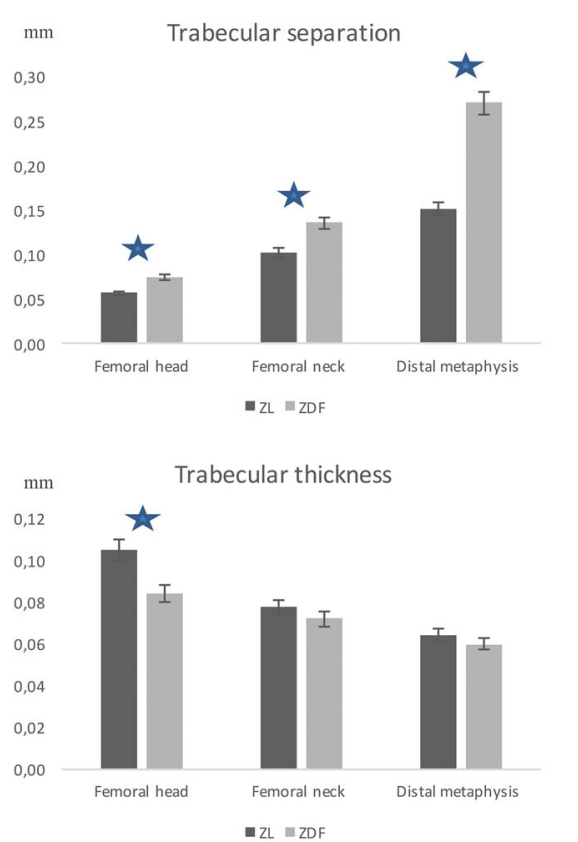

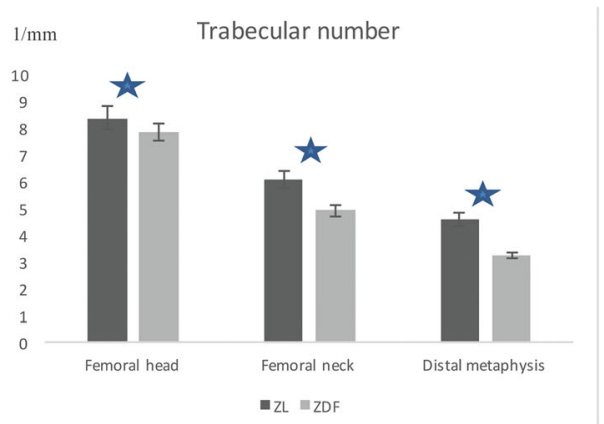

ฟ Significant differences

Fig. 2 Trabecular bone characteristics of Zucker diabetic fatty (ZDF) and Zucker lean (ZL) rats. There were significant differences between ZDF and $\mathrm{ZL}$ rats concerning the number and the separation of trabeculae in the head, neck, and distal metaphysis. Trabecular thickness was significantly different only in the head 
Table 2 Analysis of cortical bone

\begin{tabular}{llll}
\hline & ZL rats & ZDF rats & $p$ \\
\hline Femoral neck & & & \\
Ct.BV/TV (\%) & $72.80 \pm 2.96$ & $62.9 \pm 3.2$ & 0.001 \\
Ct.Ar (mm $)$ & $2.50 \pm 0.27$ & $2.23 \pm 0.12$ & 0.043 \\
Ct.Ar/Tt.Ar (\%) & $0.73 \pm 0.02$ & $0.62 \pm 0.03$ & 0.001 \\
Ct.P (mm) & $32.51 \pm 5.94$ & $31.75 \pm 2.67$ & 0.414 \\
Ct.Th (mm) & $0.15 \pm 0.03$ & $0.14 \pm 0.008$ & 0.108 \\
Ct.Po (\%) & $3.07 \pm 0.81$ & $5.15 \pm 1.77$ & 0.001 \\
vBMD & $1.21 \pm 0.07$ & $1.23 \pm 0.06$ & 0.181 \\
Femoral shaft & & & \\
Ct.BV/TV (\%) & $43.07 \pm 1.55$ & $40.09 \pm 2.17$ & 0.005 \\
Ct.Ar (mm ${ }^{2}$ ) & $5.49 \pm 0.51$ & $4.82 \pm 0.21$ & 0.005 \\
Ct.Ar/Tt.Ar (\%) & $0.43 \pm 0.01$ & $0.40 \pm 0.02$ & 0.005 \\
Ct.P (mm) & $45.87 \pm 8.13$ & $38.71 \pm 2.69$ & 0.181 \\
Ct.Th (mm) & $0.24 \pm 0.045$ & $0.25 \pm 0.025$ & 0.950 \\
Ct.Po (\%) & $3.04 \pm 0.99$ & $2.14 \pm 0.54$ & 0.05 \\
vBMD & $1.44 \pm 0.137$ & $1.39 \pm 0.09$ & 0.414 \\
\hline Data a mean 5 . & & &
\end{tabular}

Data are mean \pm standard deviation. Ct.Ar cortical area, Ct.Ar/Tt.Ar cortical area fraction, Ct.BV/TV cortical bone volume percentage, Ct.P cortical perimeter, Ct.Po cortical porosity, Ct.Th cortical thickness, $v B M D$ volumetric bone mineral density, ZDF Zucker diabetic fatty, ZL Zucker lean
Table 3 Analysis of vascularisation

\begin{tabular}{llll}
\hline & ZL rats & ZDF rats & $p$ \\
\hline Femoral neck & & & \\
W & $0.02 \pm 0.008$ & $0.012 \pm 0.002$ & 0.047 \\
W/TV (\%) & $2.8 \pm 0.55$ & $1.81 \pm 0.21$ & 0.047 \\
V.N (1/mm) & $0.64 \pm 0.07$ & $0.32 \pm 0.06$ & 0.047 \\
V.Sp (mm) & $0.25 \pm 0.02$ & $0.30 \pm 0.01$ & 0.047 \\
V.Th (mm) & $0.04 \pm 0.002$ & $0.04 \pm 0.006$ & 0.069 \\
Distal metaphysis & & & \\
W & $1.12 \pm 0.3$ & $0.44 \pm 0.11$ & 0.047 \\
W/TV (\%) & $4.35 \pm 1$ & $1.8 \pm 0.50$ & 0.047 \\
V.N (1/mm) & $0.53 \pm 0.14$ & $0.21 \pm 0.06$ & 0.047 \\
V.Sp (mm) & $0.40 \pm 0.018$ & $0.64 \pm 0.23$ & 0.047 \\
V.Th (mm) & $0.30 \pm 0.40$ & $0.08 \pm 0.009$ & 0.089 \\
\hline
\end{tabular}

Data are mean \pm standard deviation. $V . N$ vessel number, $V . S p$ vessel separation, $V$.Th vessel thickness, $W V$ vessel volume, $V V / T V$ vessel volume percentage, ZDF Zucker diabetic fatty, ZL Zucker lean

The observed lower trabecular bone volume is consistent with the results in literature reports of studies on the vertebrae $[19,20]$ and distal metaphysis of the femur [18]. In those studies, the differences observed in trabecular bone volume were explained by the number and
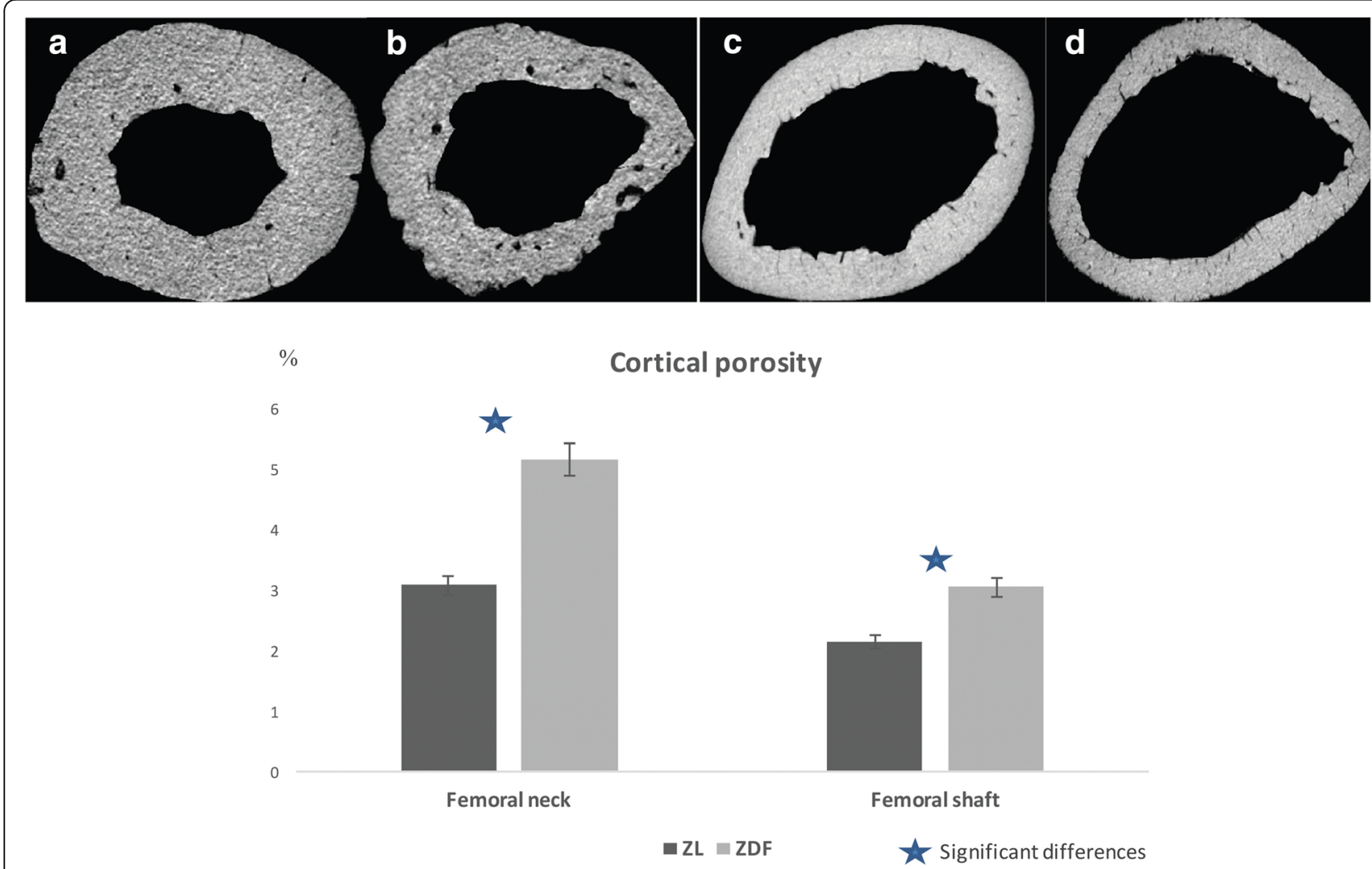

Fig. 3 Comparison of cortical porosity between a Zucker diabetic fatty (ZDF) rat and a Zucker lean ZL rat. Axial images of the femoral neck of ZL (a) and ZDF (b) rats, and the femoral shaft of ZL (c) and ZDF (d) rats. Cortical porosity was higher in ZDF compared to ZL rats, in the two locations 

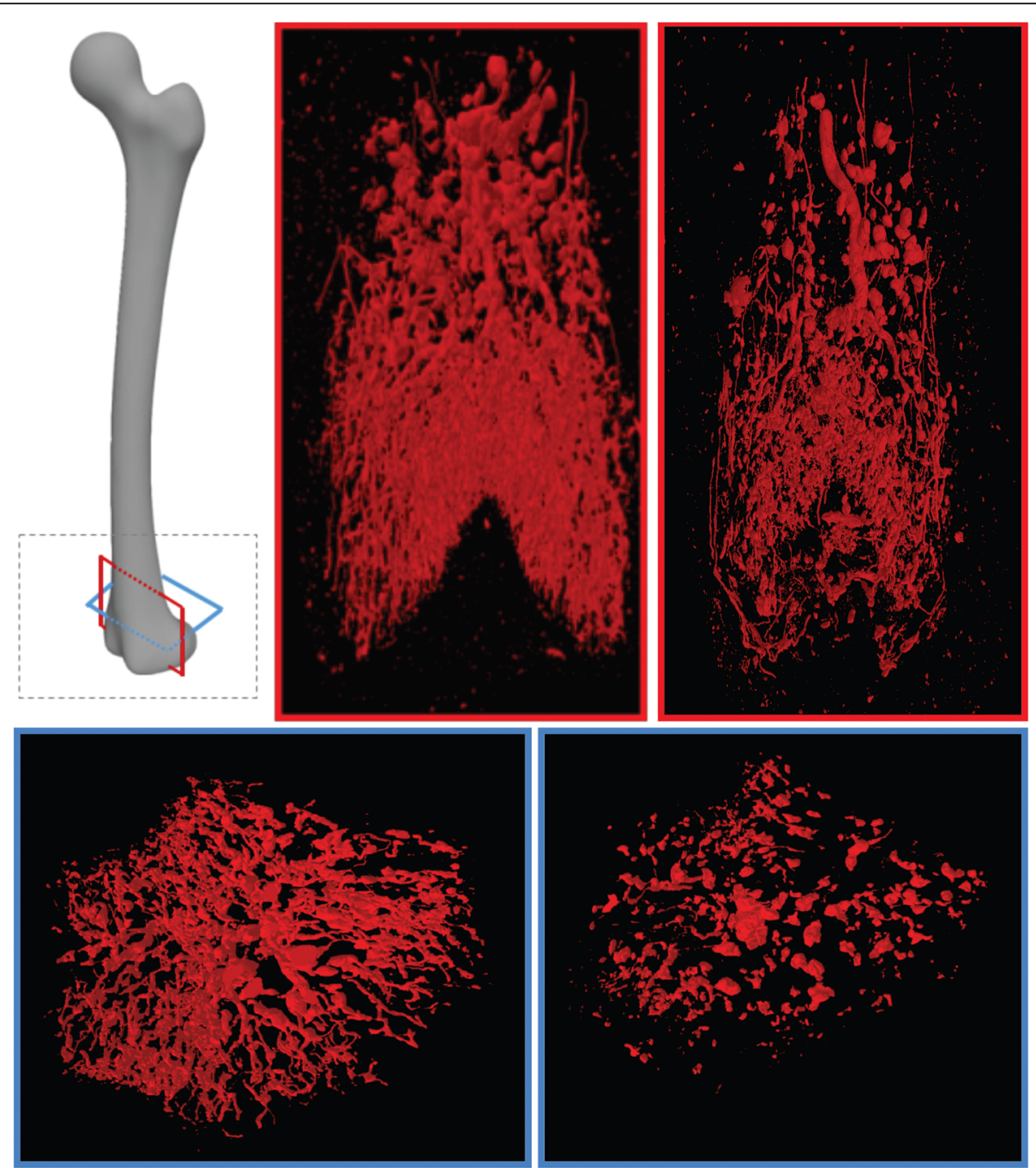

Fig. 4 Vascularisation of distal metaphysis of Zucker lean (ZL) rats and Zucker diabetic fatty (ZDF) rats, coronal and axial three-dimensional view. There was a lower vascular volume and number in ZDF rats as compared to ZL rats

thickness of trabeculae [17, 18] or thickness only [19]. These studies were carried out at resolutions of 12 and $20 \mu \mathrm{m}$ on ZDF and ZL 9- and 33-week-old rats. Our study used $6 \mu \mathrm{m}$ of resolution and 24-week-old rats. Better resolution should improve the determination of any existing differences in trabecular thickness between ZDF and ZL rats. Other studies which used ZDF rats of various ages may be needed in order to determine whether or not there is a difference.

Our differences in SMI in ZDF rats that reflect changes in the microarchitecture of trabecular bone, and may be responsible for fragility and increased fracture risk, are consistent with reports in the literature [17-19]. The papers in the literature also reported an increase in the connectivity index, indicating an anarchic reorganisation of the bony trabeculae, a result which was not observed in our study.

There is a major need to non-invasively monitor and evaluate the status of cortical bone, which plays a critical role in bone strength [14]. In this respect, mechanical testing of excised femoral necks from human cadavers has showed that the cortex contributes from 40 to $60 \%$ of the overall femoral strength [32]. Also, finite element modeling has suggested that, in the human femoral neck region, cortical bone supports $50 \%$ of the stresses associated with normal gait [33]. Moreover, administration of some newly developed antiosteoporotic drugs proved especially effective on cortical bone [34]. The mechanical properties of cortical bone depend on several structural and microstructural parameters (such as bone mineral density, cortical thickness, and cortical porosity), which can be assessed using micro-CT with appropriate spatial resolution.

Our study provided evidence of a significantly lower cortical area and of a significant increase in the cortical porosity of the neck and shaft in ZDF rats. These results are in agreement with those reported 
for the human femoral shaft in one study [19]. However, to the best of our knowledge, cortical porosity has never been studied in ZDF rats before.

Investigation of the bone microvasculature using micro-CT is challenging because vascular opacification is required for the three-dimensional study of the vascular tree [35]. Our study used information from published studies on the topic [28-30] to establish a protocol involving the use of $\mathrm{Microfil}^{\circ}$ as a contrast agent. Our procedure was complex. We judged injections as satisfactory in only 7 rats out of 12 . Such outcomes are consistent with the few reported studies that also obtained unsatisfactory opacification of small blood vessels [29]. Obtaining satisfactory $\mathrm{Microfil}^{\circ}$ injections was not the only challenge. Indeed, the CT densities of bone and opacified vessels are very similar for the two tissues and cannot be distinguished on histograms. Consequently, decalcification of the bone is mandatory. Other options were discussed in the literature. Micro-CT using synchrotron radiation offers both higher spatial resolution and higher contrast and thus provides differentiation of bone tissue from blood vessels without specimen decalcification [28] but is of limited availability. Some studies reported the use of barium sulphate for opacification of blood vessels and, thus, differentiation from bone tissue [31, 33]. Analysis of periosteal vascularisation without decalcification, using a segmentation technique, has also been reported [36] but was not appropriate for our study. Our study demonstrated that bone vascularisation in the ZDF rats was poor compared to that of the ZL rats. The number and percentage of blood vessels in the neck and distal metaphysis of the ZDF rat femurs was lower. Two femoral heads among the ZDF rats tested were unfortunately broken during manipulation, preventing measurements of the vascularisation parameters in the region of interest; in fact, the vascularisation in ZDF rats was poorer than that observed in the ZL rats. The observed difference in the vascular tissue volume may be explained by the significant difference in the number of blood vessels.

To our knowledge, there is no study regarding the bone vascular network and its correlation with alterations in bone quality in the long bones of T2DM rats. Our study applied micro-CT analysis to study bone vasculature and showed impaired bone vascularisation in ZDF rats. It should be noted that the observed vascular changes were more pronounced at the distal metaphysis of the femur where major bone quantity and quality changes were observed. The underlying mechanisms for these events remain unclear. Several hypotheses such as the role of vascularisation in bone remodelling units through complex molecular communications and recruitment of bone cells from pericytes [30], or vasomotor alterations of the principal nutrient artery of the femur from ZDF rats [37], have been proposed. It is therefore possible that the microangiopathy may be at least in part responsible for the bone microarchitecture changes observed in diabetic subjects.

The present study has limitations. First, the sample size dedicated to the study of bone vessels in the femur of rats using micro-CT was quite small. Indeed, we obtained satisfactory opacification of the vessels in only seven out of 12 rats. Second, our analysis was conducted using a resolution of $6 \mu \mathrm{m}$, so that an accurate identification of pores was difficult as regards to pore size which varies between 5 and $0.1 \mathrm{~mm}$. The use of a better resolution would probably allow for more accurate analysis of the porosity of bone in ZDF rats. Anyway, the results observed in our study are consistent with literature reports of an increased in porosity in men with T2DM $[14,38,25]$.

In summary, our study using micro-CT provided evidence that, firstly, trabecular and cortical bone quantity and microarchitecture were altered in the femur of ZDF rats; secondly, bone vasculature was altered in the femur of ZDF rats; and, thirdly, the main region of interest for studying architecture and vasculature changes in the femur of ZDF rats was the distal metaphysis. Our preliminary study regarding bone vascularisation needs confirmation with larger populations.

\section{Abbreviations \\ Conn.D: Index of connectivity; Ct.Ar: Cortical surface; Ct.BV/TV: Cortical bone percentage; Ct.P: Cortical perimeter; Ct.Po: Cortical porosity; Ct.Th: Cortical thickness; micro-CT: Microcomputed tomography; SMI: Structural model index; T2DM: Type 2 diabetes mellitus; Tb.BV: Trabecular bone volume; Tb.BV/ TV: Trabecular bone percentage; Tb.N: Trabecular number; Tb.Sp: Trabecular separation; Tb.Th: Trabecular thickness; V.N: Vessel number; V.Sp: Vessel separation; V.Th: Vessel thickness; VBMD: Volumetric bone mineral density; VOI: Volumes of interest; W: Vessel volume; W/TV: Vessel percentage; ZDF: Zucker diabetic fatty; ZL: Zucker lean}

\section{Funding}

This study was supported by the "Fondation de I'Avenir" (ET3-694), and the Institut Français pour la Recherche Odontologique (IFRO).

\section{Availability of data and materials}

The datasets generated and/or analysed during the current study are not publicly available due to ongoing analyses but are available from the corresponding author on reasonable request.

\section{Authors' contributions}

VB and FA conceived and coordinated the study. FA and GC performed the injection and recovered the femurs. DZ, VB, MS, and JMC chose the imaging protocols. DZ and MS gathered all the micro- $C T$ data and made the measurements. Statistical analysis was performed by VB and DZ. VB and DZ also drafted the manuscript with the help of FA and JMC. All authors read and approved the final manuscript.

Ethics approval and consent to participate

Ethics Committee of the University Paris Diderot (N01610.01/S69), Paris, France.

Consent for publication

Not applicable.

Competing interests

The authors declare that they have no competing interests. 


\section{Publisher's Note}

Springer Nature remains neutral with regard to jurisdictional claims in published maps and institutional affiliations.

\section{Author details \\ 'Centre hospitalier Lariboisière, Hopital Lariboisière, Service de radiologie ostéo-articulaire, 2 rue Ambroise Paré, 75010 Paris, France. ${ }^{2}$ CNRS Laboratoire B2OA, Laboratoire B2OA.10, Avenue de Verdun, 75010 Paris, France. ${ }^{3}$ Centre hospitalier Amiens, Chu Amiens, Service de radiologie, Chemin de Longpré, 80080 Amiens, France.}

\section{Received: 3 September 2018 Accepted: 28 February 2019}

Published online: 11 April 2019

\section{References}

1. Wild S, Roglic G, Green A, Sicree R, King H (2004) Global prevalence of diabetes. Diabetes Care 27:1047-1053

2. Schwartz AV (2016) Epidemiology of fractures in type 2 diabetes. Bone 82: 2-801 /03/2019 21:40:00

3. Leslie WD, Rubin MR, Schwartz AV, Kanis JA (2012) Type 2 diabetes and bone. J Bone Miner Res 27:2231-2237

4. Bonds DE, Larson JC, Schwartz AV et al (2006) Risk of fracture in women with type 2 diabetes: the women's health initiative observational study. J Clin Endocrinol Metab 91:3404-3410

5. Vestergaard P (2006) Discrepancies in bone mineral density and fracture risk in patients with type 1 and type 2 diabetes - a meta-analysis. Osteoporos Int 18:427-444

6. Melton LJ 3rd, Riggs BL, Leibson CL et al (2008) A bone structural basis for fracture risk in diabetes. J Clin Endocrinol Metab 93:4804-4809

7. Janghorbani M, Dam RMV, Willett WC, Hu FB (2007) Systematic review of type 1 and type 2 diabetes mellitus and risk of fracture. Am J Epidemiol 166:495-505

8. Yamamoto M, Yamaguchi T, Yamauchi M, Kaji H, Sugimoto T (2009) Diabetic patients have an increased risk of vertebral fractures independent of BMD or diabetic complications. J Bone Miner Res 24:702-709

9. Semel J, Gray JM, Ahn HJ, Nasr H, Chen JJ (2010) Predictors of outcome following hip fracture rehabilitation. PM R 2:799-805

10. Reistetter TA, Graham JE, Deutsch A, Markello SJ, Granger CV, Ottenbacher KJ (2011) Diabetes comorbidity and age influence rehabilitation outcomes after hip fracture. Diabetes Care 34:1375-1377

11. Hernandez RK, Do TP, Critchlow CW, Dent RE, Jick SS (2012) Patient-related risk factors for fracture-healing complications in the United Kingdom General Practice Research Database. Acta Orthop 83:653-660

12. Ma L, Oei L, Jiang L et al (2012) Association between bone mineral density and type 2 diabetes mellitus: a meta-analysis of observational studies. Eur J Epidemiol 27:319-332

13. Deandrea S, Lucenteforte E, Bravi F, Foschi R, La Vecchia C, Negri E (2010) Risk factors for falls in community-dwelling older people: a systematic review and meta-analysis. Epidemiology 21:658-668

14. Burghardt AJ, Issever AS, Schwartz AV et al (2010) High-resolution peripheral quantitative computed tomographic imaging of cortical and trabecular bone microarchitecture in patients with type 2 diabetes mellitus. J Clin Endocrinol Metab 95:5045-5055

15. Farr JN, Drake MT, Amin S, Melton LJ 3rd, McCready LK, Khosla S (2014) In vivo assessment of bone quality in postmenopausal women with type 2 diabetes. J Bone Miner Res 29:787-795

16. Patsch JM, Li X, Baum T et al (2013) Bone marrow fat composition as a novel imaging biomarker in postmenopausal women with prevalent fragility fractures. J Bone Miner Res 28:1721-1728

17. Hamann C, Goettsch C, Mettelsiefen J et al (2011) Delayed bone regeneration and low bone mass in a rat model of insulin-resistant type 2 diabetes mellitus is due to impaired osteoblast function. Am J Physiol Endocrinol Metab 301:E1220-E1228

18. Hamann C, Rauner M, Höhna Y et al (2013) Sclerostin antibody treatment improves bone mass, bone strength, and bone defect regeneration in rats with type 2 diabetes mellitus. J Bone Miner Res 28:627-638

19. Reinwald S, Peterson RG, Allen MR, Burr DB (2009) Skeletal changes associated with the onset of type 2 diabetes in the ZDF and ZDSD rodent models. Am J Physiol Endocrinol Metab 296:E765-E774

20. Mathey J, Horcajada-Molteni MN, Chanteranne B et al (2002) Bone mass in obese diabetic Zucker rats: influence of treadmill running. Calcif Tissue Int 70:305-311
21. Prisby RD, Swift JM, Bloomfield SA, Hogan HA, Delp MD (2008) Altered bone mass, geometry and mechanical properties during the development and progression of type 2 diabetes in the Zucker diabetic fatty rat. J Endocrinol 199:379-388

22. Karim L, Bouxsein ML (2016) Effect of type 2 diabetes-related non-enzymatic glycation on bone biomechanical properties. Bone 82:21-27

23. Karim L, Tang SY, Sroga GE, Vashishth D (2013) Differences in nonenzymatic glycation and collagen cross-links between human cortical and cancellous bone. Osteoporos Int 24:2441-2447

24. Karim L, Vashishth D (2012) Heterogeneous glycation of cancellous bone and its association with bone quality and fragility. PLoS One 7:e35047

25. Shu A, Yin MT, Stein E et al (2012) Bone structure and turnover in type 2 diabetes mellitus. Osteoporos Int 23:635-641

26. Starup-Linde J, Vestergaard P (2016) Biochemical bone turnover markers in diabetes mellitus: a systematic review. Bone 82:69-78

27. García-Martín A, Rozas-Moreno P, Reyes-García R et al (2011) Circulating levels of sclerostin are increased in patients with type 2 diabetes mellitus. J Clin Endocrinol Metab 97:234-241

28. Duvall CL, Taylor WR, Weiss D, Gulberg RE (2004) Quantitative microcomputed tomography analysis of collateral vessel development after ischemic injury. Am J Physiol Heart Circ Physiol 287:H302-H310

29. Roche B, David V, Vanden-Bossche A et al (2012) Structure and quantification of microvascularisation within mouse long bones: what and how should we measure? Bone 50:390-399

30. Lafage-Proust MH, Roche B, Langer M et al (2015) Assessment of bone vascularization and its role in bone remodeling. Bonekey Rep 4:662

31. Fei J, Peyrin F, Malaval L, Vico L, Lafage-Proust MH (2010) Imaging and quantitative assessment of long bone vascularization in the adult rat using microcomputed tomography. Anat Rec (Hoboken) 293:215-224

32. Werner C, Iversen BF, Therkildsen MH et al (1988) Contribution of the trabecular component to mechanical strength and bone mineral content of the femoral neck. An experimental study on cadaver bones. Scand J Clin Lab Invest 48:457-460

33. Lotz JC, Cheal EJ, Hayes WC et al (1995) Stress distributions within the proximal femur during gait and falls: implications for osteoporotic fractures. Osteoporos Int 5:252-261

34. Hyldstrup L, Conway GS, Racz K et al (2012) Growth hormone effects on cortical bone dimensions in young adults with childhood-onset growth hormone deficiency. Osteoporosis Int 23:2219-2226

35. Nyangoga $H$, Mercier P, Libouban H, Baslé MF, Chappard D (2011) Threedimensional characterization of the vascular bed in bone metastasis of the rat by microcomputed tomography (MicroCT). PLoS One 6:e17336

36. Engelke K, Karolczak M, Lutz A, Seibert U, Schaller S, Kalender W (1999) Micro-CT technology and application for assessing bone structure Radiologe 39:203-212

37. Stabley JN, Prisby RD, Behnke BJ, Delp MD (2015) Type 2 diabetes alters bone and marrow blood flow and vascular control mechanisms in the ZDF rat. J Endocrinol 225:47-58

38. Farr JN, Khosla S (2016) Determinants of bone strength and quality in diabetes mellitus in humans. Bone $82 \cdot 28$-34

\section{Submit your manuscript to a SpringerOpen ${ }^{\circ}$ journal and benefit from:}

- Convenient online submission

- Rigorous peer review

- Open access: articles freely available online

- High visibility within the field

Retaining the copyright to your article

Submit your next manuscript at $>$ springeropen.com 